\title{
Effective Conductivity of Two-Dimensional Heterostructures with Non-Screened Potential
}

\author{
F. T. Vasko ${ }^{1,2}$ and G.-Q. Hai ${ }^{1}$ \\ ${ }^{1}$ Instituto de Física de São Carlos, Universidade de São Paulo, 13560-970 São Carlos, SP, Brazil \\ ${ }^{2}$ Institute of Semiconductor Physics, NAS, 45 Pr. Nauky, Kiev, 03650, Ukraine
}

Received on 23 April, 2001

\begin{abstract}
The effective magnetoconductivity tensor of selectively doped heterostructures with non-screened potential is evaluated for high-frequency regime. The lateral inhomogeneity of the donor distribution in the structure results in a large-scale variation of the energy levels. The contribution due to such inhomogeneity takes place in addition to the standard Drude mechanism of absorption and this mechanism is dominant for the high-frequency regime.
\end{abstract}

\section{Introduction}

In high-frequency regime, if the photon frequency $\omega$ or the cyclotron frequency $\omega_{c}$ exceed the relaxation frequency (but corresponding energies are smaller than the mean electron energy), the linear response of electrons shows the Drude dispersion, i.e. the conductivity is found to be proportional to $\omega^{-2}$ or $\omega_{c}^{-2}$. This general dependency appears to be violated in semiconductor heterostructures with non-screened potential. The screening is not effective on a random potential due interface roughness or due to in-plane non-uniform $\delta$ doping in QW with two occupied subbands[1] or in multiple selectively doped QWs[2].

In the present paper, we describe the response of the 2D high-mobility electrons in selectively doped heterostructures taking into account an in-plane electron redistribution under non-screened random potential due to lateral inhomogeneity of $\delta$-doping (see Fig.1). Based on an electrostatic description of the non-screened variation of the potential, the effective conductivity tensor of the non-uniform electron gas is obtained from the linearized kinetic equation in Sec. II. The interplay between the Drude frequency dispersion, due to the standard relaxation processes, and the additional contribution, due to the planar large-scale inhomogeneity, is described in Sec. III. This frequency dependency of the effective conductivity is analyzed for the case of acoustic phonon scattering. Our numerical results demonstrate an essential modification on the response. The concluding remarks are presented in the last section.

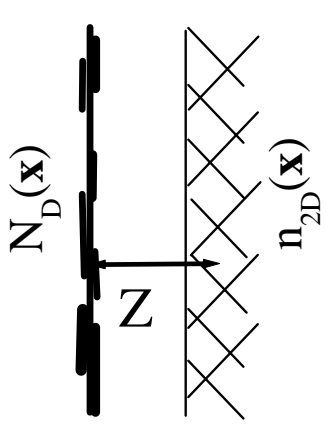

(a)

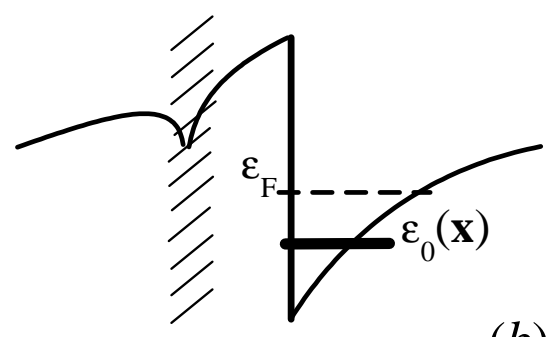

(b)

Figure 1. Schematic view of (a) the heterojunction with $\delta$ doping $N_{2 D}(\mathbf{x})$ and (b) the band diagram along $z$ axis, with non-uniform ground level $\varepsilon_{o}(\mathbf{x})$.

\section{Effective Conductivity Tensor}

The self-consistent description of the $2 \mathrm{D}$ electrons in selectively-doped structures with large-scale variations of donor distribution over the $2 \mathrm{D}$ plane is based on the Schrödinger and the Poisson equations. Within the first order approximation to the inhomogeneous potential $w_{\mathbf{x}}$, the electron concentration $n_{\mathbf{x} z}$ can be written in the form $n_{\mathbf{x} z}=\rho_{2 D}\left(\varepsilon_{F}-w_{\mathbf{x}}\right) \psi_{z}^{2}$, where $\rho_{2 D}$ is the $2 \mathrm{D}$ density of states, $\varepsilon_{F}$ the Fermi energy, and $\psi_{z}$ the wavefunction of the ground level. For the large-scale 
variation of the potential (under the condition $l_{c} \gg a_{B}$, where $l_{c}$ is the lateral correlation length of non-uniform donor distribution and $a_{B}$ is the Borh radius), the solution of the Poisson equation is of a simple form[2]:

$$
w_{\mathbf{q}} \simeq-\frac{\delta n_{\mathbf{q}}}{\rho_{2 D}} e^{-q Z}
$$

where $\delta n_{\mathbf{q}}$ is the Fourier component of the $2 \mathrm{D}$ donor concentration and $Z$ is the average distance between the $2 \mathrm{D}$ electrons and the $\delta$-layer. Thus, the non-screened potential is suppressed if $Z \gg l_{c}$. For further numerical estimations we assume a Gaussian correlation of variation of the donor concentration. Within the second order accuracy, the correlation function $W(q)=$ $\int d(\Delta \mathbf{x}) \exp (-i \mathbf{q} \cdot \Delta \mathbf{x})\left\langle w_{\mathbf{x}} w_{\mathbf{x}^{\prime}}\right\rangle$ is given by

$$
W(q)=\pi l_{c}^{2}\left(\frac{\overline{\delta n}}{\rho_{2 D}}\right)^{2} \exp \left[-\left(q l_{c} / 2\right)^{2}-2 q Z\right],
$$

where $\overline{\delta n}$ is the typical variation of the $2 \mathrm{D}$ concentration and $n_{2 D}=\rho_{2 D} \varepsilon_{F}$.

In a lateral electric field $\mathbf{E}_{\mathbf{x}} \exp (-i \omega t)$ and a transverse magnetic field $\mathbf{H} \| O Z$, the linear response of the $2 \mathrm{D}$ electrons within the dispersion law $\varepsilon_{p \mathbf{x}}=\varepsilon_{p}+w_{\mathbf{x}}$ $\left(\varepsilon_{p}=p^{2} / 2 m\right.$ is the kinetic energy and $m$ is the effective mass) is described by the field-induced contribution to the distribution function: $\left(\phi_{\varepsilon \mathbf{q}}+\chi_{\mathbf{p q}}\right) \exp (-i \omega t+i \mathbf{q x})$. Separating the symmetric and non-symmetric part of the distribution function, $\phi_{\varepsilon \mathbf{x}}$ and $\chi_{\mathbf{p q}}$, respectively, we write the linearized kinetic equation as follows (see [3] for details):

$$
\begin{gathered}
\left(i \omega+\widehat{J}_{n e l}\right) \phi_{\varepsilon \mathbf{q}}=i\left\{(\mathbf{q} \cdot \mathbf{v}) \chi_{\mathbf{p q}}\right\} \\
\widehat{\mathcal{R}}_{\mathbf{q}} \chi_{\mathbf{p q}}-i\left\{(\mathbf{q} \cdot \mathbf{v}) \chi_{\mathbf{p q}}\right\}=\Pi_{\mathbf{p q}}-i(\mathbf{q} \cdot \mathbf{v}) \phi_{\varepsilon \mathbf{q}}
\end{gathered}
$$

Here $\widehat{J}_{n e l}$ is the non-elastic collision integral, $\mathbf{v}=\mathbf{p} / m$, and $\{\cdots\} \equiv \int_{0}^{2 \pi} d \varphi(\cdots) /(2 \pi)$ stands for the average over the in-plane angle $\varphi$. The collisionless evolution operator $\widehat{\mathcal{R}}_{\mathbf{q}}$ and the non-homogeneous term $\Pi_{\mathbf{p q}}$ are given by

$$
\widehat{\mathcal{R}}_{\mathbf{q}}=\left\{i(\mathbf{q} \cdot \mathbf{v}-\omega)+\nu_{e}+\left[\boldsymbol{\omega}_{c} \times \mathbf{p}\right] \cdot \nabla_{\mathbf{p}}\right\}^{-1},
$$

and

$$
\Pi_{\mathbf{p q}}=\sum_{\mathbf{q}^{\prime}}\left(e \mathbf{E}_{\mathbf{q}^{\prime}} \cdot \mathbf{v}\right) \int \frac{d \mathbf{x}}{L^{2}} e^{-i\left(\mathbf{q}-\mathbf{q}^{\prime}\right) \mathbf{x}} \delta\left(\varepsilon_{F \mathbf{x}}-\varepsilon_{p}\right),
$$

respectively, where $\nu_{e}$ is the momentum relaxation frequency corresponding to the short range scattering mechanism, $\boldsymbol{\omega}_{c}=|e| \mathbf{H} /(m c)$ the cyclotron frequency, $\mathbf{E}_{\mathbf{q}}$ the Fourier component of the electric field, and $\varepsilon_{F \mathbf{x}}=\varepsilon_{F}+w_{\mathbf{x}}$ the non-uniform Fermi energy. The non-symmetric part of the distribution is determined by Eq. (4) and can be expressed through

$$
\left\{(\mathbf{q} \cdot \mathbf{v}) \chi_{\mathbf{p q}}\right\}=\frac{\left\{(\mathbf{q} \cdot \mathbf{v}) \widehat{\mathcal{R}}_{\mathbf{q}} \Pi_{\mathbf{p q}}\right\}-\left\{(\mathbf{q} \cdot \mathbf{v}) \widehat{\mathcal{R}}_{\mathbf{q}}(\mathbf{q} \cdot \mathbf{v})\right\} \phi_{\varepsilon \mathbf{q}}}{1-i\left\{(\mathbf{q} \cdot \mathbf{v}) \widehat{\mathcal{R}}_{\mathbf{q}}\right\}}
$$

The symmetric part of distribution is given by $\phi_{\varepsilon \mathbf{q}} \simeq$ $\left\{(\mathbf{q} \cdot \mathbf{v}) \chi_{\mathbf{p q}}\right\} / \omega$ for the high-frequency limit.

The Fourier components of the induced charge $\rho_{\mathbf{q}}$ and current $\mathbf{j}_{\mathbf{q}}$ are introduced through the standard relations: $\rho_{\mathbf{q}}=\left(2 e^{2} / L^{2}\right) \sum_{\mathbf{p}} \phi_{\varepsilon \mathbf{q}}$, and $\mathbf{j}_{\mathbf{q}}=$ $\left(2 e^{2} / L^{2}\right) \sum_{\mathbf{p}} \mathbf{v} \chi_{\mathbf{p q}}$. The average induced current is expressed through $\left\langle\phi_{\varepsilon \mathbf{q}}\right\rangle$ and $\left\langle\Pi_{\mathbf{p q}}\right\rangle$, where $\langle\cdots\rangle$ stands for the average over the random potential. Since the average values are proportional to $\delta_{\mathbf{q} 0}$, we obtain $\left\langle\mathbf{j}_{\mathbf{q}}\right\rangle=\delta_{\mathbf{q} 0}$ j. After the summation over $\mathbf{p}$ we transform the induced current into

$$
\mathbf{j}=\frac{e^{2}}{2 m L^{2}} \sum_{\mathbf{q}}\left[i\left\langle n_{-\mathbf{q}} \mathbf{E}_{\mathbf{q}}\right\rangle k_{\omega}^{(+)}+\frac{\boldsymbol{\omega}_{c} \times\left\langle n_{-\mathbf{q}} \mathbf{E}_{\mathbf{q}}\right\rangle}{\omega_{c}} k_{\omega}^{(-)}\right]
$$

with

$$
k_{\omega}^{( \pm)}=\left(\frac{1}{\omega+\omega_{c}+i \nu_{e}} \pm \frac{1}{\omega-\omega_{c}+i \nu_{e}}\right)
$$

where the right side is written through the Fourier component of the concentration $n_{\mathbf{q}}=n_{2 D}+\rho_{2 D} w_{\mathbf{q}}$. Separating the uniform and potential parts of electric field (under the condition $\operatorname{curl} \mathbf{E}_{\mathbf{x}}=0$ ), we write $\mathbf{E}_{\mathbf{q}}$ as $\mathbf{E}_{\mathbf{q}}=\mathbf{E} \delta_{\mathbf{q} 0}-i \mathbf{q} \Phi_{\mathbf{q}}$ with $\mathbf{E}$ the homogeneous electric field. The random Fourier component of the potential $\Phi_{\mathbf{q}}$ is determined from the Poisson equation with the high-frequency charge density $\rho_{\mathbf{q}}$. Within the first order approximation to the random contribution, its solution appears to be proportional to $(\mathbf{q} \cdot \mathbf{E}) w_{\mathbf{q}}$. Substituting the random field into Eq. (5) and averaging according to Eq. (2), we obtain $\mathbf{j}$ through the effective conductivity tensor as $\mathbf{j}=\widehat{\sigma}_{\omega}^{e f f} \mathbf{E}$. The diagonal (non-diagonal) component of the conductivity $\sigma_{d}^{e f f}\left(\sigma_{\perp}^{e f f}\right)$ is written as

$$
\sigma_{d, \perp}^{e f f}=\sigma_{d, \perp}\left[1+L^{-2} \sum_{\mathbf{q}} \frac{W(q)}{\varepsilon_{F}} \mathcal{A}_{d, \perp}(q)\right]
$$

where $\sigma_{d, \perp}$ corresponds to the uniform case. Since $\mathcal{A}_{d}(q)$ and $\mathcal{A}_{\perp}(q)$ depend on $\omega$ and $\omega_{c}$, the above equation describes the frequency dispersion of the effective magnetoconductivity (including the case of cyclotron resonance). It also appears to be temperature dependent through the relaxation frequency $\nu_{e}$. A geneal expression for $\sigma_{d, \perp}^{e f f}$ may be obtained after the evaluation of $\mathcal{A}_{d, \perp}(q)$ and the integration over $\mathbf{q}$ in Eq. (6).

\section{Spectral Dependencies}

We consider now, in the absence of magnetic field, the modification on the Drude spectral dependency $\sigma_{\omega}^{e f f}$ result from the second addendum to Eq. (6). Using an explicit expression for $\Phi_{\mathbf{q}}$ and performing the average 
over non-screened random potential, we transform the non-uniform contribution as follows:

$$
\mathcal{A}_{d}(q)=-\frac{\left(d \mathcal{B}_{\varepsilon q} / d \varepsilon\right)_{\varepsilon_{F}}}{a_{B} q+\mathcal{B}_{\varepsilon q}}
$$

with

$$
\mathcal{B}_{\varepsilon q}=\left\{\frac{\mathbf{q} \cdot \mathbf{v}}{\mathbf{q} \cdot \mathbf{v}-\omega-i \nu_{e}}\right\}\left(1+\left\{\frac{i \nu_{e}}{\mathbf{q} \cdot \mathbf{v}-\omega-i \nu_{e}}\right\}\right)^{-1} .
$$

Further, performing the integral over $\mathbf{q}$ we find the effective conductivity in the form $\sigma_{\omega}^{e f f}=$ $\left(e^{2} n_{2 D} / m \nu_{e}\right) F(\Omega, \eta)$ with the dimensionless function:

$$
\begin{gathered}
F(\Omega, \eta)=\frac{i}{\Omega+i}\left[1+\frac{\Omega}{2}\left(\frac{\overline{\delta n}}{n_{2 D}} \eta\right)^{2}\right. \\
\left.\times \int_{0}^{\infty} \frac{d x \exp \left(-x^{2}-\gamma x\right) x^{3}}{V_{\Omega}(\eta x)\left[V_{\Omega}(\eta x)-i\right]\left[(1+\lambda x)\left(V_{\Omega}(\eta x)-i\right)-\Omega\right]}\right],
\end{gathered}
$$

where $\Omega=\omega / \nu_{e}, \eta=2 l_{F} / l_{c}, \gamma=4 Z / l_{c}, \lambda=a_{B} / l_{c}$, and $V_{\Omega}(\eta x)=\sqrt{(\Omega+i)^{2}-(\eta x)^{2}}$.

The numerical calculation is performed for $\delta$-doped AlGaAs/GaAs heterojunction with $\overline{\delta n} / n_{2 D} \approx 0.3$. We have used $\nu_{e}^{-1} \approx 76 \mathrm{ps}$ and $l_{F} \approx 14.7 \mu \mathrm{m}$ in agreement with the experimental data[4] for the structures with high mobility. Figure 2 shows the frequency dependence of $\operatorname{Re} F(\Omega, \eta)$ for different $\eta$ and $\gamma$. The parameter $\lambda$ is determined through $\eta$ by $\lambda=\left(a_{B} / 2 l_{F}\right) \eta$. The Drude dispersion for the ideal structure is given by the dotted curve in the figure. One can see that the random-induced absorption is comparable (or up to 10 times greater) to the Drude contribution and the hump-type dispersion takes place for the high-frequency region. The considered frequency corresponds to $\mathrm{GHz}$ frequency range.

\section{Conclusion}

In this work, we have studied the effective conductivity tensor of the selectively-doped heterostructures with non-screened large-scale random potential induced by the inhomogeneity of the doping layer. The presence of such a potential leads to a lateral variation of the energy levels and, consequently, modifies qualitatively the character of the frequency response of the system due to the contribution of non-uniform electric field. This new mechanism of the frequency dispersion is essential for the spectral region $\omega \gtrsim v_{F} / l_{c}$ and the obtained contribution is proportional to the mean square variation of the level in the weak disorder regime. Since the Drude absorption is suppressed as $\omega^{-2}$ at high frequency, the proposed mechanism appears to be a few times greater than the standard result (see Fig.2 ).

To conclude, the obtained spectral (or temperature) dependencies of effective conductivity will stimulate an experimental study of high-frequency response taking into account the lateral inhomogeneity of selectivelydoped structures. Along with the frequency measurements of electron response, other characteristics of nonhomogeneous structures, like 2D plasmon dispersion or cyclotron resonance, are also influenced by the nonscreened variation of the levels. In addition, the temperature dependence of mobility and the static magnetotransport phenomena should be modified due to the analogous mechanism. In the static case we need to find $\phi_{\varepsilon \mathbf{q}}$ taking into account the non-elastic collisions. These phenomena require a special consideration.

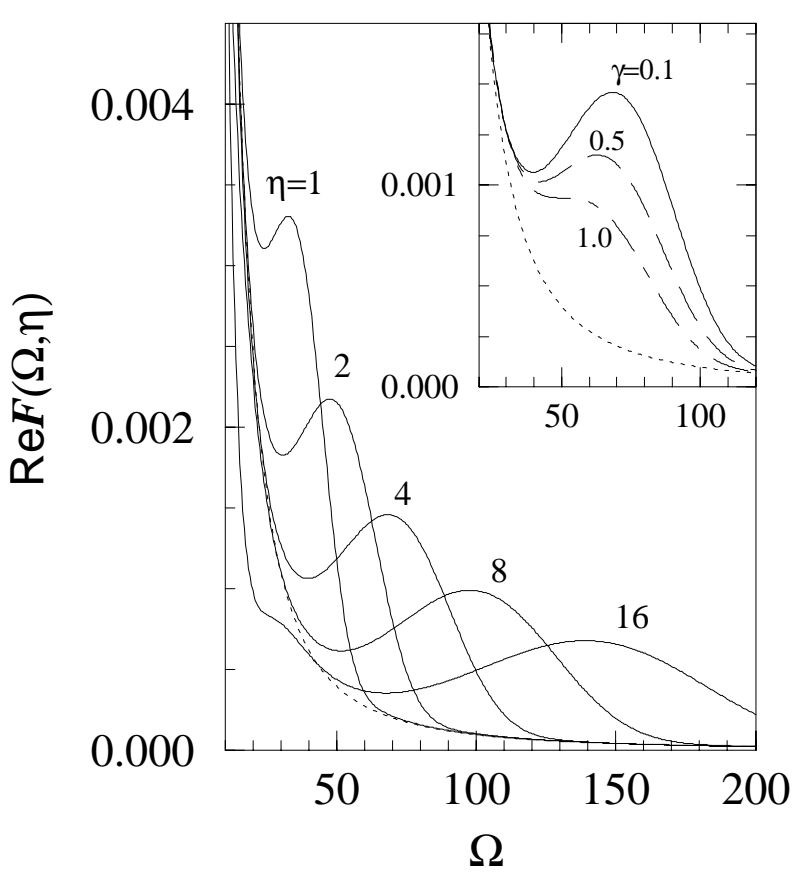

Figure 2. The real part of $\sigma_{\omega}^{e f f}$ versus the dimensionless frequency $\Omega=\omega / \nu_{e}$ at $\gamma=0.1$ for different $\eta=2 l_{F} / l_{c}$. Inset shows $\operatorname{Re} F(\Omega, \eta)$ for different $\gamma=4 Z / l_{c}$ at $\eta=4$.

\section{Acknowledgments}

This work was supported by FAPESP and CNPq (Brazilian agencies).

\section{References}

[1] O.G. Balev, F.T. Vasko, F. Aristone, and N. Studart, Phys. Rev. B 62, 10212 (2000); F.T. Vasko, O.G. Balev and N. Studart, Phys. Rev. B 62, 12940 (2000).

[2] F.T. Vasko, and G.-Q. Hai, Phys. Rev. B, submitted.

[3] E.M. Lifshitz and L.P. Pitaevskii, Physical Kinetics, Pergamon Press, Oxford (1981).

[4] P.J. Burke, I.B. Spielman, J.P. Eisenstein, L.N. Pfeiffer, and K.W. West. Appl. Phys. Lett. 76, 745 (2000). 